\title{
Hallazgos neuro-estrabismológicos en una paciente con síndrome de Kabuki
}

\author{
Neuro-ophthalmology and strabismus findings in a patient with Kabuki \\ syndrome
}

\author{
Martín Gallegos-Duarte ${ }^{1}$ ，Isidro Gutiérrez-Álvarez ${ }^{2} \quad$, Danjela Ibrahimi ${ }^{3} \quad$, Isidro Gutiérrez-Villaseñor ${ }^{4}$ \\ Citación: Gallegos-Duarte M, Gutiérrez-Álvarez I, Ibrahimi D, Gutiérrez-Villaseñor I. Hallazgos neuro-estrabismológicos en una paciente con \\ Síndrome de Kabuki. Ustasalud 2020;19: 42-48.
}

Licencia Creative Commons

\section{(c) (1) () ())} alterarlos, adicionalmente se debe reconocer la autoría de las personas que figuran en las publicaciones, pero estas no podrán ser comercializadas.

\section{Resumen}

El síndrome de Kabuki es un trastorno congénito, evolutivo y permanente que tiene manifestaciones clínicas características. Se presenta el caso de una niña con diagnóstico de síndrome de Kabuki MLL2 positivo, a quien se le realizó tipificación, caracterización clínica, estudio oftalmológico, estrabismoglógico, análisis perceptual visual, optométrico y estudios de neuroimagen. Se identificaron facies característica, estrabismo disociado, anisocoria, disminución de habilidades perceptuales, hipocoherencia y asimetría interhemisférica importante para Delta y Theta, disminución del volumen del hipocampo izquierdo y de la arborización de la vía visual en su porción posterior izquierda, disminución de la colina y un incremento del lactato cortical. El estudio reveló alteraciones corticales y de la vía visual.

Palabras clave: Corteza cerebral, estrabismo, neuroimagen.

\section{Abstract}

Kabuki syndrome is a congenital, evolutionary, and permanent disorder that has characteristic clinical manifestations. The case of a girl with a diagnosis of MLL2 positive Kabuki syndrome is presented who underwent typing, clinical characterization, ophthalmological study, strabismological, visual perceptual analysis, optometric and neuroimaging studies. Characteristic facies, dissociated strabismus, anisocoria, decreased perceptual abilities, hypo coherence and significant interhemispheric asymmetry were identified for Delta and Theta, decreased volume of the left hippocampus and arborization of the visual pathway in its left posterior portion, decreased choline and an increase in cortical lactate. The study revealed cortical and visual pathway alterations.

Keywords: Cerebral cortex, strabismus, neuroimaging.
1 Doctor en Investigación Médica. Licenciatura en Optometría, Facultad de Medicina, Universidad Autónoma de Querétaro, Querétaro (México).

2 Médico especialista. Licenciatura en Optometría, Facultad de Medicina, Universidad Autónoma de Querétaro, Querétaro (México).

3 Doctora en Optometría. Licenciatura en Optometría, Facultad de Medicina, Universidad Autónoma de Querétaro, Querétaro (México).

4 Médico especialista. Instituto Mexicano de Seguro Social (IMSS), León (México).

Autor de correspondencia:

Martín Gallegos Duarte

Correo electrónico:

martin.gallegos@uaq.mx 


\section{INTRODUCCIÓN}

El síndrome de Kabuki es un trastorno congénito, evolutivo y permanente que manifiesta un abanico de alteraciones clínicas características. La etiología del síndrome no es del todo conocida, pero se han reportado anormalidades citogenéticas, tales como microdeleción cromosómica y duplicación submicroscópica. Se sabe que el patrón de herencia es autosómico dominante y que la mayoría de los casos reportados son esporádicos, lo que sugiere que se trata de mutaciones de novo ${ }^{1,2}$.

Pese a que existen patrones que harían pensar en una herencia familiar, aún se sigue la línea del gen MLL2 (KBUK1), esto es, se mantiene la etiología citogénetica por mutación heterocigota en el cromosoma 12q12-q14 del gen MLL2(KMT2D), por mutación en el cromosoma Xp11.3 del gen KDM6A KABUK1 y KABUK2 en OMIM, en concordancia con lo encontrado citogenéticamente con el síndrome de Kabuki ${ }^{3,4}$.

Este trastorno fue descrito inicialmente en Japón en 1981 por Niikawa y colaboradores y Kuroki y colaboradores, de ahí la denominación de "Síndrome de Niikawa-Kuroki”, pero fue Niikawa quien le adjudicó el nombre de "make-up- maquillaje Kabuki", en alusión al tipo de maquillaje usado por los actores del teatro tradicional japonés ${ }^{5-7}$.

Mientras que en 1988, Niikawa describió 62 casos, integrando el diagnóstico con base en cinco características principales: alteraciones faciales, anomalías esqueléticas, alteración en dermatoglifos, retraso mental y talla baja. Matsumoto categorizó el síndrome en criterios mayores y menores. Dentro de los criterios mayores se encuentran: fisuras palpebrales largas con eversión del párpado inferior, puente nasal deprimido, cejas arqueadas, almohadillas en el pulpejo de los dedos, pabellones auriculares prominentes o malformados, acortamiento del quinto dedo, paladar alto o hendido, dentadura anormal, hipotonía, retraso mental, talla baja, ptosis e hipoacusia ${ }^{5}$.

Por su parte, los siguientes rasgos se consideran como criterios menores: escleras azules, escoliosis, anomalías cardiovasculares, malformaciones renales, vértebras deformadas, criptorquidia y deficiencia de la hormona del crecimiento ${ }^{5}$.
Entre las anomalías más sobresalientes reportadas en la literatura y que caracterizan la expresión fenotípica del síndrome de Kabuki, se encuentran las anomalías faciales, tales como: fisura palpebral elongada, eversión del tercio lateral del párpado inferior, cejas arqueadas con tercio lateral despoblado, raíz nasal ancha con punta deprimida, pabellones auriculares grandes y dismórficos. Entre las anomalías faciales que se han encontrado, destacan: paladar ojival, fisura palatina, micrognatia, dentición anormal, raíz de implantación del cabello baja, fosita/fístula o apéndice preauricular ${ }^{2}$.

Se han descrito distintas anomalías neurológicas, tales como hipotonía, convulsiones y microcefalia, así como retraso mental leve o moderado. Se han encontrado, además, anomalías esqueléticas, del crecimiento, vertebrales, braquidactilia del quinto dedo, coxa valga, luxación congénita de cadera, luxación de rodilla, epífisis en cono, cardiopatía congénita, coartación de la aorta, mal posición renal, hipoplasia renal, displasia renal y criptorquidia. Hasta aquí, las alteraciones más citadas en la literatura ${ }^{5}$.

La red de centros de especialización para dismorfología (DYSCERNE) continúa desarrollando pautas diagnósticas y terapéuticas para el síndrome de Kabu$\mathrm{ki}^{8} \mathrm{y}$, aunque la prevalencia es baja, ya que se estima entre $1 / 86.000$ y $1 / 320.000$ nacidos vivos de ambos sexos, se han descrito más de 400 casos en todo el mundo y cada vez se encuentran nuevos casos en una amplia variedad de nacionalidades y etnias. Estudios recientes revelan un porcentaje cada vez más elevado de anomalías que involucran los sistemas: nervioso, renal, urogenital y cardiovascular ${ }^{5,9}$.

Actualmente, se considera que la presencia de dimorfismos faciales, presentes en el $94 \%$ de los casos, así como las dificultades de alimentación y la hipotonía, que están presentes en todos los casos reportados en el estudio de DYSCERNE, constituyen hallazgos que sugieren el diagnóstico clínico de síndrome de Kabuki².

Las alteraciones oftalmológicas, aunque esporádicas, están relacionadas con el síndrome. Kluijt y colaboradores reportaron ambliopía, errores de refracción, estrabismo, nistagmo, colobomas, micro-córnea, opacidades corneales, escleróticas azules, cataratas, obstrucción naso-lacrimal y un posible lipoma caruncular ${ }^{10}$. 
Todo lo anterior se ha reportado en la literatura ya, sin embargo, hasta ahora no se habían descrito las alteraciones estrabismológicas, visuoperceptuales y corticales del síndrome de Kabuki de manera concomitante, ni se había hecho una correlación clínico-neuroimagenológi$\mathrm{ca}$, que es el motivo del presente estudio.

\section{REPORTE DE CASO}

Se trata de una niña de seis años y medio de edad, oriunda de Querétaro, México. Madre de 34 años, cuatro embarazos, dos abortos, es la hija mayor. Sin antecedentes de consanguinidad. Embarazo de 39 semanas que cursó con polihidramnios, peso al nacer $3.850 \mathrm{~g}$. Presentó dificultad respiratoria y neumonía al nacer. $\mathrm{Su}$ desarrollo ha mostrado talla baja, retraso psicomotor moderado, sin cuadros infecciosos ni convulsivos, tampoco se identificaron alteración auditiva ni hormonales.

El diagnóstico de síndrome de Kabuki MLL-2 positivo fue realizado por un servicio de genética externo, mientras que en nuestro servicio se le realizaron los siguientes procedimientos: estudio optométrico, oftalmológico, análisis perceptual visual, así como pupilometría en condiciones escotópicas con luz infrarroja por parte de nuestro equipo de investigación.

El estudio optométrico incluyó agudeza visual, retinoscopia con y sin cicloplejia. El estudio oftalmológico incluyó la valoración del segmento anterior, reflejos pupilares, fondo de ojo y la motricidad ocular.

Por su parte, el estudio perceptual incluyó pruebas de habilidades visuales (TPS), pruebas de discriminación auditiva-visual, visual-escritura, escuchar-escribir y visual-oral, prueba de memoria visual de Monroe, así como prueba de discriminación auditiva-visual (VADS).

Se hizo electroencefalograma (EEG) interpolado a Mapeo Cerebral Digitalizado (MCD) y análisis neurométrico, por un neurofisiólogo externo y, finalmente, se realizaron estudios de resonancia magnética (RMI) incluida una Tractografía de la vía visual, así como la espectroscopía de resonancia magnética en un gabinete especializado.

Se encontraron los siguientes hallazgos generales: talla corta, hiperelasticidad cutánea y retraso mental moderado.
Hallazgos faciales: dismorfia facial caracterizada por puente nasal ancho, orejas grandes, boca, manos pequeñas, almohadillas en los pulpejos.

Entre los hallazgos oftalmológicos más destacados se encontró telecanto, euribléfaron, cejas arqueadas y pobladas, escleróticas azules, estéreo-ciega, agudeza visual 20/30 en isovisión, exotropia de ángulo variable, Desviación Horizontal Disociada, síndrome en "V", hiperfunción de músculos oblicuos inferiores, patrón orbitario anti mongoloide, astigmatismo hipermetrópico $\mathrm{y}$ anisocoria de $1.1964 \mathrm{~mm}(10,4 \%)$ por miosis en el ojo derecho en condiciones escotópicas (Figuras 1, 2 y 3 ).

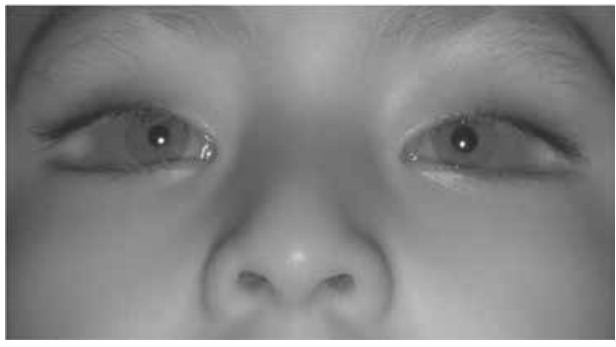

Figura 1. Fotografía clínica tomada mediante técnica infrarroja en condiciones escotópicas. Muestra puente nasal ancho, telecanto, euribléfaron, cejas arqueadas y pobladas, así como anisocoria.

\begin{tabular}{|c|c|c|}
\hline \multicolumn{3}{|c|}{ MIDAIASIS } \\
\hline & Der & $\mathrm{Izq}$ \\
\hline \multicolumn{3}{|l|}{ Córnea } \\
\hline Area $=$ & $54950.00000 \mathrm{C}$ & $54892.00000 \mathrm{C}$ \\
\hline Perimetro= & 830.951294 & 830.951294 \\
\hline Diámetro $=$ & 262.000000 & 256.000000 \\
\hline \multicolumn{3}{|l|}{ Pupila } \\
\hline Area $=$ & $10753.00000 \mathrm{C}$ & $15839.00000 \mathrm{C}$ \\
\hline Perímetro= & 367.566315 & 446.106201 \\
\hline Diámetro= & 115.000000 & 139.000000 \\
\hline Razón= & $43.89 \%$ & $54.30 \%$ \\
\hline Dif. entre pupilas= & $1.1964 \mathrm{~mm}$ & $10.40 \%$ \\
\hline Anisocoria= & $\mathrm{Si}$ & \\
\hline
\end{tabular}

Figura 2. Mediante análisis pupilométrico se determinó el área, al perímetro y el diámetro de cada pupila, encontrando una anisocoria de $1,1964 \mathrm{~mm}$ equivalente $10,4 \%$ de diferencia en el tamaño pupilar, en condiciones escotópicas.

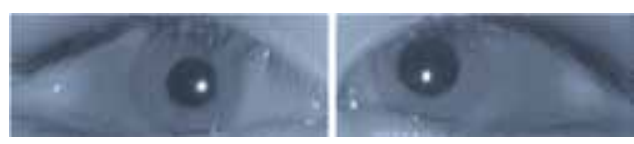

Figura 3. Fotografía clínica tomada mediante técnica infrarroja en condiciones escotópicas. Muestra una anisocoria que se manifiesta en condiciones escotópicas, debido a la exacerbación del reflejo constrictor de la pupila del ojo derecho. 
El examen estrabismoglógico mostró exotropia de ángulo variable de entre orto y -20 dioptrías prismáticas, así como falta de estereopsis acorde a la prueba de Lang, por lo que se sospechó que se tratase de una Desviación Horizontal Disociada, lo que indicaría una mayor alteración neuronal respecto a los estrabismos no disociados ${ }^{11-13}$.

Por su parte, el análisis perceptual mostró una especial dificultad para la lecto escritura. Un retraso en el tiempo durante el seguimiento vertical y horizontal. Fue notoria además la dificultad para la coordinación fina ojo-mano. La paciente mostró una edad menor que la cronológica para la percepción visual, mientras que la prueba de inversión de Gardner reveló dificultades para comprender el concepto de imagen en espejo y la reversibilidad, además de que estuvo fuera de los límites para su edad en el reconocimiento visual de letras y números.

La prueba de habilidades visuales (TVPS) mostró una edad de cuatro años para memoria visual, discriminación visual y de 4,10 años en memoria secuencial. La prueba de memoria visual de Monroe indicó una edad de cinco años. La prueba de discriminación auditiva-visual (VADS) expuso un desempeño bajo, siendo la habilidad visual-escritura la más baja, mientras que las pruebas de escuchar-escribir, escuchar-oral y visual-oral obtuvieron un nivel medio.

La refracción encontrada bajo cicloplejia fue OD: $+1,25=-0,75 \times 180^{\circ}$ y para OS: $+0,75=-0,25 \times 0^{0}$ logrando una agudeza visual estimada de $20 / 40$ para cada ojo. Por su parte, la prueba de Lang para estéreo agudeza fue negativa.

El Mapeo cerebral mostró asimetría intrahemisférica para Delta y Alfa en regiones frontales derechas, para Delta y Theta en P4-T6, O2-T6 y T4-T6. T5-T6 mostró una asimetría interhemisférica importante.

El análisis neurométrico mostró hipocoherencia interhemisférica importante para Delta, Theta y Alfa en F1-F2, hipocoherencia Delta en F3-F4 y F7-F8, Hipercoherencia Alfa y Beta en P3-P4, y Beta para T5-T6. Además, se encontró hipercoherencia interhemisférica para Delta, Theta, Alfa y Beta en C3-P3 y C3-O1, así como múltiples zonas de hipercoherencia distribuida en ambos hemisferios y en todas las bandas frecuenciales. Se encontró fase Lag intrahemisfé- rica en P3-T5 para Teta y O1-T5 para Theta y Alfa. El principal disturbio de la fase Lag se encontró en C3-C4 para Teta, F7-F8 para Delta y en T5-T6 para Delta, Teta y Beta.

La Tractografía de la vía visual mostró una disminución del volumen del hipocampo izquierdo $\left(501,1 \mathrm{~mm}^{2}\right)$ en relación con el derecho $\left(524,1 \mathrm{~mm}^{2}\right)$ (Figura 4). Este estudio de neuroimagen mostró además una asimetría en la arborización del tracto visual en su parte distal hacia los lóbulos occipitales, lo cual sugiere una disminución de la conectividad neuronal hacia la vía visual izquierda (Figura 5).

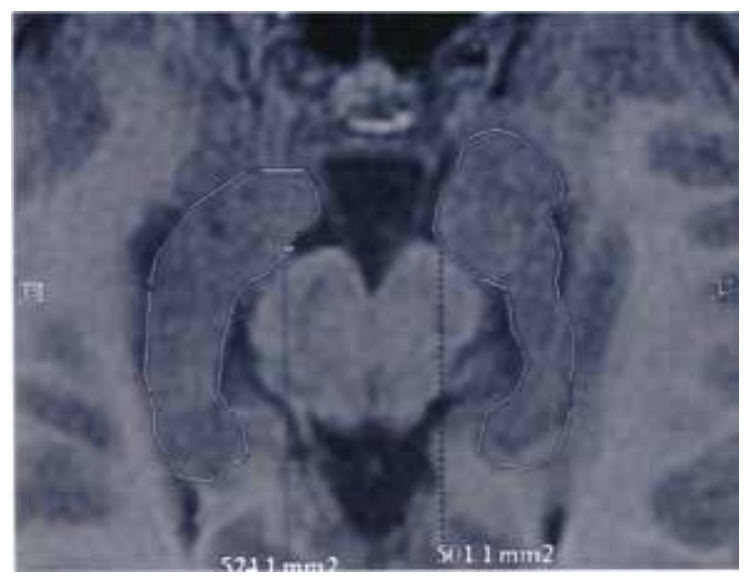

Figura 4. Estudio de Resonancia Magnética. Muestra una disminución en el tamaño del hipocampo izquierdo.

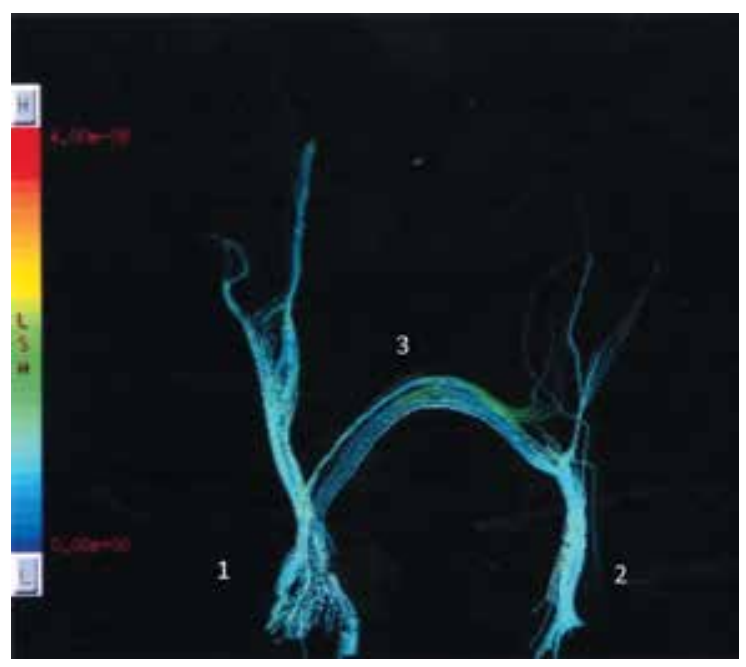

Figura 5. La Tractografía de la sustancia blanca de la vía visual derecha en su porción posterior muestra una mayor arborización (1) que el lado izquierdo (2) mientras que el Splenium (3) que es determinante para la intercomunicación entre la vía visual izquierda y derecha, se muestra anatómicamente íntegro. 
La Espectroscopía de Resonancia Magnética reveló una disminución importante de la Colina (Co), de 0,45 U así como un incremento del Lactato, lo que constituye el hallazgo neurometabólico más sobresaliente (Figura 6).

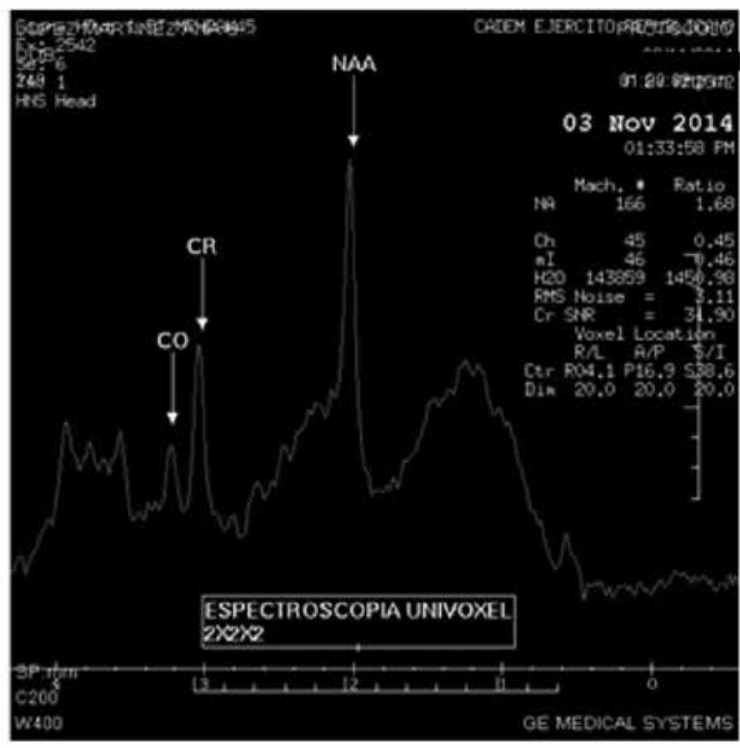

Figura 6. La espectroscopía de RMI muestra disminución de la colina (Co) y aumento del lactato, mientras e N- Actilaspartato (NAA) permaneció dentro de límites normales.

\section{DISCUSIÓN}

La prevalencia de este síndrome es baja, pero ha sido motivo de estudio y se han publicado una serie de artículos que destacan las alteraciones cromosómicas, así como las manifestaciones clínicas de este, sin embargo, es la primera vez que se describen los hallazgos estrabismológicos, corticales y perceptuales, realizándose una correlación clínica con alteraciones en los estudios de neuroimagen.

Es importante recordar que Pascual-Castroviejo en 1985 analizó 18 casos de síndrome de Kabuki implementando algunos estudios de neuroimagen, tales como EEG, líquido cefalorraquídeo, neumoencefalografía, tomografía computarizada y resonancia magnética cerebral; sin embargo, a diferencia de nuestro estudio, sus reportes de neuroimagen dieron resultados negativos, esto es, fueron reportados como "normales"13.

El caso ahora presentado mostró alteraciones neuroeléctricas corticales demostradas mediante
Neurometría y Mapeo Cerebral Digitalizado, siendo la hipocoherencia en las porciones anteriores de la corteza cerebral lo más destacado.

Por su parte, el análisis neurometabólico indicó, por un lado, una evidente disminución de la colina y por otro, un incremento del lactato, situación que no es usual14. Lo anterior es importante toda vez que la colina está particularmente implicada en los circuitos de la memoria y la recompensa, por lo que una disminución podría explicar el bajo desempeño tanto de la memoria visual, como de la habilidad visual-escritura.

Los hallazgos encontrados e integrados a partir del análisis perceptual, la pupilometría y los estudios de neuroimagen sugieren un daño cortical temprano durante la corticogénesis. Con base en lo anterior, es plausible afirmar que los estudios visuoperceptuales realizados, interpretados y contextualizados adecuadamente pueden ser un método clínico efectivo para sospechar que existen alteraciones corticales.

Un punto importante para destacar fue el comportamiento pupilar en condiciones escotópicas. En este sentido, llamó la atención la evidente anisocoria debido a una pobre dilatación del ojo izquierdo en condiciones escotópicas, lo cual indica que existe una exacerbación del reflejo constrictor de la pupila, esto es, prevalece el reflejo constrictor de la pupila. Este hallazgo sugiere que la corteza cerebral cuando está afectada puede perder el control de este reflejo. Es plausible afirmar entonces, que la prevalencia del reflejo constrictor sobre el dilatador indica una diferencia filogénica, en donde el reflejo constrictor es más elaborado y delicado que el dilatador, así, las alteraciones corticales encontradas en la paciente, junto con la evidente anisocoria fortalecen la tesis que cuando el integrador central falla, los reflejos oculomotores se exacerban tal y como propone el neuroestrabismo ${ }^{15}$.

Respecto a los cambios neuroanatómicos evidenciados mediante resonancia magnética, llama la atención, la disminución del volumen del hipocampo izquierdo (Figura 4), así como la pobre arborización de la vía visual en su porción posterior izquierda que muestra la Tractografía (Figura 5). Estos hallazgos indican que existe una disminución en la conecti- 
vidad y están acorde con el reporte neuroeléctrico y neurometabólico, lo que podría explicar el bajo rendimiento perceptual visual de la paciente.

Las porciones posteriores del cerebro fueron las más afectadas anatómicamente, sin embargo, las porciones anteriores resultaron afectadas funcionalmente, tanto las coherencias como el reflejo pupilar. Esto se explica por la disposición anatómica del reflejo pupilar, que posee una relación anatómica distinta, siendo anterior respecto al hipocampo y la corteza cerebral. Del mismo modo, pese a la alteración pupilar izquierda, el estudio perceptual visual mostró que el ojo izquierdo fue el ojo dominante motor y sensorial.

Desde un punto de vista perceptual, tanto la memoria visual como la discriminación auditiva visual y visual-escribir, resultaron bajas, lo que significa que la paciente presenta dificultad para la lecto-escritura. Mientras que el retraso en el tiempo de seguimiento vertical y horizontal observado en la paciente sugiere dificultades en la automatización oculomotora.

Es importante realizar un abordaje interdisciplinario en los pacientes con síndrome de Kabuki. Entre los estudios que deberían considerarse para su inclusión proponemos el estudio estrabismoglógico, la pupilometría, el análisis perceptual visual, así como el uso de neuroimagen, a fin de evaluar la función cortical en pacientes con este síndrome.

\section{Consideraciones éticas}

Se cuenta con la autorización escrita por parte de la madre de la paciente, para la publicación del presente informe, incluyendo las fotografías clínicas y de neuroimagen.

\section{REFERENCIAS}

1. Hernández-Woodbine MJ, Del Castillo-Rix DS, Baquero-Mejía IC. Reporte de una nueva mutación en Colombia: un paciente con síndrome de Kabuki. Iatreia 2019;33(1):78-83. doi: 10.17533/udea.iatreia.38.

2. Dentici ML, Di Pede A, Lepri FR, Gnazzo M, Lombardi $\mathrm{MH}$, Auriti C, et al. Kabuki syndrome: clini- cal and molecular diagnosis in the first year of life. Arch Dis Child. 2015;100(2):158-64. DOI: 10.1136/ archdischild-2013-305858.

3. Banka S, Veeramachaneni R, Reardon W, Howard E, Bunstone S, Ragge N, et al. How genetically heterogeneous is Kabuki syndrome?: MLL2 testing in 116 patients, review and analyses of mutation and phenotypic spectrum. Eur J Hum Genet. 2012 Apr;20(4):381-8. DOI: 10.1038/ejhg.2011.220.

4. Miyake N, Koshimizu E, Okamoto N, Mizuno S, Ogata T, Nagai T, et al. MLL2 and KDM6A mutations in patients with Kabuki syndrome. Am J Med Genet A. 2013 Sep;161A(9):2234-43. DOI: 10.1002/ajmg.a.36072.

5. Suárez Guerrero JL, Ordónez Suárez AA, Contreras García GA. Síndrome de Kabuki. Ann Pediatr (Barc). 2012;77(1):51-6. DOI: 10.1016/j.anpedi.2012.01.016.

6. Barradas-Hernández M, Velasco-Orozco M, Jardines-Serralde L. Hallazgos audiológicos en el síndrome Kabuki. Reporte de un caso. Rev Mex AMCAOF 2015;4(1):23-31.

7. Osorio-Alarcón C, Olaya-Mantilla C, Silvera-Redondo C, Garavito-Galofre P. Síndrome de Kabuki: presentación de un caso y revisión de la literatura. Revista Científica Salud Uninorte 2016;32(3):565-75.

8. Griffiths P, Strong K, Gardner S, et al. DYSCERNE: developing clinical management guidelines for selected dysmorphic syndromes. Orphanet J Rare Dis. 2010;5(Suppl 1): P20. DOI:10.1186/1750-1172-5-S1-P20.

9. Caciolo C, Alfieri P, Piccini G, Digilio MC, Lepri FR, Tartaglia M, et al. Neurobehavioral features in individuals with Kabuki syndrome. Mol Genet Genomic Med. 2018 May;6(3):322-331. DOI: 10.1002/mgg3.348.

10. Kluijt I, Van Dorp DB, Kwee ML, Toutain A, Keppler-Noreuil K, Warburg M. Kabuki syndrome - report of 6 cases and review of the literature with emphasis on ocular features. Ophthalmol Genet 2000; 21: 51-61. DOI: 10.1076/1381-6810(200003)2111-IFT051.

11. Evans SL, Kumar N, Rashid MH, Hughes DS. New ocular findings in a case of Kabuki syndrome. Eye (Lond). 2004 Mar;18(3):322-4. DOI: 10.1038/sj.eye.6700649.

12. Gallegos-Duarte M, Mendiola-Santibanez JD, Ibrahimi D, Paredes-Orta C, Rodríguez-Resendiz J, González-Gutiérrez CA. A novel method for measuring subtle alterations in pupil size in children with congenital strabismus. IEEE Access 2016;4(1):1-14. DOI: 10.1109/ ACCESS.2020.3007873.

13. Pascual Castroviejo I, Pascual Pascual SI, Velázquez Fragua R, Palencia Luaces R. Síndrome del maquillaje Kabuki. A propósito de 18 casos españoles. Rev Neurol 2005;40(8):473-78. Doi: 10.33588/rn.4008.2004521. 
14. Fitzgerald BA, Billson FA. Dissociated vertical deviation: evidence of abnormal visual pathway projection. Br J Ophthalmol. 1984;68(11):801-6. DOI: 10.1136/ bjo.68.11.801.
15. Aksoy DÖ, Alkan A. Neurometabolic Diseases in Children: Magnetic Resonance Imaging and Magnetic Resonance Spectroscopy Features. Curr Med Imaging Rev. 2019;15(3):255-268. DOI: 10.2174/157340561366 6171123152451 .

\section{Correos electrónicos de los autores:}

Martín Gallegos-Duarte: martin.gallegos@uaq.mx

Isidro Gutiérrez-Álvarez: yagayagayo@hotmail.com

Danjela Ibrahimi: danel.ibrahimi@uaq.mx

Isidro Gutiérrez-Villaseñor: iso.exu@gmail.com 